\title{
On the Coderivative of the Projection Operator onto the Second-order Cone
}

\author{
Jiří V. Outrata $\cdot$ Defeng Sun
}

Published online: 13 June 2009

(C) Springer Science + Business Media B.V. 2009

\section{Erratum to: Set-Valued Anal (2008) 16: 999-1014 DOI 10.1007/s11228-008-0092-x}

In the original article a mistake has been introduced into the abstract. To put matters right we are now reprinting the abstract as it should have appeared:

The limiting (Mordukhovich) coderivative of the metric projection onto the second-order cone in $\mathbb{R}^{n}$ is computed. This result is used to obtain a sufficient condition for the Aubin property of the solution map of a parameterized secondorder cone complementarity problem and to derive necessary optimality conditions for a mathematical program with a second-order cone complementarity problem among the constraints.

Everything else in the paper remains correct.

The online version of the original article can be found at http://dx.doi.org/10.1007/s11228-008-0092-x.

J. V. Outrata $(\varangle)$

Institute of Information Theory and Automation,

Academy of Sciences of the Czech Republic,

18208 Prague, Czech Republic

e-mail: outrata@utia.cas.cz

D. Sun

Department of Mathematics and Risk Management Institute,

National University of Singapore, 2 Science Drive 2,

Kent Ridge, Republic of Singapore

e-mail: matsundf@nus.edu.sg 